\title{
Characterization of Non-Structural Gene of Influenza A Virus H1N1 in Iran
}

\author{
Zahra Asadollahi' $^{1}$ D, Farida Behzadian' ${ }^{1}$ Fatemeh Fotouhi ${ }^{2}$, Behrokh Farahmand ${ }^{2 *}$ (D), Samaneh Kouhestani ${ }^{1}$
}

1. Biosciences and Biotechnology Research Center, Malek-Ashtar University of Technology,, Tehran, Iran.

2. Department of Influenza and Other Respiratory Viruses, Pasteur Institute of Iran, Tehran, Iran.

\begin{tabular}{c|c}
$\begin{array}{c}\text { Use yur devic to scan } \\
\text { and read the article online }\end{array}$ \\
Influenza A Virus H1N1 in Iran. Research in Molecular Medicine. 2019; 7(2):1-8. https://doi.org/10.32598/rmm.7.2.51 \\
Ifol https://doi.org/10.32598/rmm.7.2.51
\end{tabular}

\section{(i) $\$$}

Article Type:

Research Paper

Article info:

Received: 26 Feb 2019

Revised: 21 Apr 2019

Accepted: 15 Mar 2019

Keywords:

Influenza A (H1N1), NS

segment, Phylogenetic

analysis, Protein structure

\section{A B S T RACT}

Background: The Non-Structural (NS) genomic segment of influenza A virus expresses two proteins (NS1 and NS2) responsible for virulence and pathogenicity. Here, we characterize the NS gene of H1N1 influenza viruses isolated from Iranian patients during the 2015 and 2017 outbreaks.

Materials and Methods: Influenza A positive specimens with high viral load were selected for virus amplification on MDCK (Madin-Darby Canine Kidney) cells to obtain sufficient viral RNA for RT-PCR. The NS segments were amplified and sequenced from randomly selected samples. Genetic characterization, phylogenetic, and protein modeling analyses were carried out using Bioedit, MEGA7 software, and Muster, Modweb, Modfold, Tm-align web servers. Reference sequences from other geographic regions were available on GenBank.

Results: Phylogenetic analysis on the NS gene of A/H1N1 isolates revealed that all Iranian isolates, except the A/Tehran/SMO08/2015, were close to strains from Continental countries of Asia and North America, with 99\% identity. Meanwhile the mentioned exception was clustered with some strains from Iran, Kazakhstan, Myanmar, Turkmenistan, and Jordan. Overall six substitutions were observed through the deduced Iranian NS1 and NS2 amino acid sequences. One of the mutations (the E96D mutation from the isolate A/Tehran/A7106/2017) was first observed in the present project. Prediction of three-dimensional structure of the Iranian NS1 and NS2 proteins in comparison with counterpart references available in PDB showed a slight deviation in the functional domains. However, the high similarity of the Iranian NS gene with those from other countries indicate no significant changes in the molecular features of the NS genes.

Conclusion: The severity of the symptoms does not seem to be caused by the observed mutations. Molecular analysis of other alleles is needed to explain the high pathogenic feature of the 2015 isolates.

\section{Introduction}

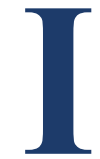

nfluenza viruses are responsible for acute respiratory infection, being a source of seasonal epidemics and occasional pandemics [1].
Influenza A viruses are enveloped viruses of the family Orthomyxoviridae. They are further classified into subtypes depending upon their surface glycoproteins, Haemagglutinin (HA) and Neuraminidase (NA). Influenza A viruses contain a single-stranded, negative-sense,

\section{* Corresponding Author:}

Behrokh Farahmand, PhD.

Address: Department of Influenza and Other Respiratory Viruses, Pasteur Institute of Iran, Tehran, Iran.

Phone: +98 (21)64112183

E-mail: b_farahmand@pasteurac.ir 
segmented RNA genome consisting of eight segments that encode 11 known proteins $[2,3]$. To restrict virus proliferation, virus-infected cells usually mount a potent and diverse antiviral response. Thus, to survive in nature, influenza A viruses has evolved multiple mechanisms to circumvent these defenses. Some strategies are strainspecific, such as increased replication speed or decreased sensitivity to host-cell antiviral effectors $[4,5]$.

Segment 8 of influenza A(H1N1) encodes two proteins: NS1 (nonstructural) protein and NS2 or NEP (nuclear export protein) by alternative splicing. The mRNAs of both proteins share 56 nucleotides at the 5 ' end, resulting in both proteins sharing 10 amino acids at N-terminal. NS1 protein is encoded by the collinear mRNA from segment 8 of the influenza virus genome and has a strain specific length ranging from 230-237 amino acid residues. The NS1 protein comprises two functional domains: the Nterminal (amino acids 1 to 73) RNA-Binding Domain (RBD) and the C-terminal (amino acids 74 to 230/237) Effector Domain (ED), which binds several cellular proteins and is proposed to bind nuclear proteins to carry out NS1 functions in vivo [6, 7].

NS1 is a multifunctional protein involved in various functions of regulating immune responses. It functions as an Interferon (IFN) antagonist, which allows efficient virus replication in IFN-competent hosts by targeting both IFN $/ \alpha / \beta$ production and the activation of IFN-induced antiviral genes [8]. NS1 binds directly to the regulatory subunit of Protein Kinase R (PKR), regulating the effectors of IFN response and controlling apoptosis, cell growth, cell proliferation, cytokine production, and signaling [9]. In addition, NS1 protein is also involved in inhibiting 3 ' end processing of host mRNA by binding to CPSF30 (cleavage and polyadenylation specificity factor 30) and PABPN1 (poly (A) binding protein nuclear 1) [9]

Considering the important role of NS proteins to surpass the cellular innate immune response, regulate influenza virus transcription and replication, and act as a virulence determinant in influenza infection, we characterized the NS gene segments of influenza A (H1N1) viruses randomly isolated from Iranian patients during the 2015 deadly flu outbreak and the 2017 seasonal outbreak. The analyses performed were phylogenetic topology, genetic clustering, pairwise amino acid variations and $3 \mathrm{D}$ protein structural modeling.

\section{Materials and Methods}

\section{Clinical case definition and Virus isolation}

Influenza viruses were isolated by standard methods from nasal swabs of patients with clinical symptoms at the Pasteur Institute of Iran, during fall and winter of 2015 and 2017. The samples had been tested by a realtime RT-PCR assay for detection and subtype identification. High titer H1N1 samples were randomly selected and propagated on MDCK (Madin-Darby Canine Kidney) cells.

\section{Amplification and sequencing of the NS gene}

Viral RNA was extracted from $200 \mu \mathrm{L}$ of the cell culture supernatant for each sample using the "High pure

Table 1. Primers sequences used for amplification and sequencing of the NS full length fragment

\begin{tabular}{cc}
\hline Property & Direction \\
\hline $5^{\prime}$-AGCAAAAGCAGGGTGACAAA - $3^{\prime}$ & Forward \\
$5^{\prime}$-AGTAGAAACAAGGGTGTTTT - 3' & Reverse \\
\hline \$RMm
\end{tabular}

Table 2. PCR parameters

\begin{tabular}{cccc}
\hline Number of Cycles & Step & Temperature $^{\circ} \mathrm{C}$ & Time \\
\hline & Initial denaturation & 94 & $2 \mathrm{~min}$ \\
& Denaturation & 94 & $15 \mathrm{sec}$ \\
30 Cycles & Annealing & 50 & $30 \mathrm{sec}$ \\
& Extension & 68 & $50 \mathrm{sec}$ \\
& Final extension & 68 & $50 \mathrm{sec}$ \\
\end{tabular}


viral RNA kit" (Roche, Germany) and eluted in $20 \mu \mathrm{L}$ DEPC treated water. Specific primers for amplification of the target sequence ( $890 \mathrm{nt}$ of NS segment), were designed by E. Hoffman et al. (Table 1) [10]. The primers were controlled with the Gene Runner software.

The PCR reaction was carried out according to Table 2. PCR products were gel-purified using GF-1 PCR Clean-up Kit (Vivantis, Malaysia) based on manufacturer's instruction and subjected to sequencing at the Sequence Laboratories of First Base company, Malaysia. The sequences were edited and assembled in Chromas Lite version 2.5.1 (Technelysium Pty Ltd., Unit 406, 8 Cordelia St., South Brisbane QLD 4101, Australia) and CLC sequence viewer V.6.7. The nucleotide sequences of all samples were deposited in the GenBank database under the accession numbers shown in Table 3.

Multiple sequence alignment and phylogenetic analysis

Evolutionary analyses based on NS1 gene sequence was carried out by including the globally representative A/H1N1pdm09 sequence from the NCBI Influenza Resource Database (http://www.ncbi.nlm.nih.gov/ genomes/FLU). All NS gene sequences were aligned using the ClustalW alignment tool in the Bioedit software v. 7.2.5. Phylogenetic trees were constructed by the Neighbor-joining method [11] inferred based on the best fit nucleotide substitution model for the NS genes as implemented in MEGA 7.0.

\section{D protein structure modeling and superimposition}

Modeling the 3D structure of the NS1 and NS2 proteins was done using the Modweb (https://modbase. compbio.ucsf.edu/modweb) server [12]. Previously published structures of the NS1 effector domain (PDBID 3M5R) and of the NS2 (PDBID 1PD3) were selected as templates for comparative modeling. Each model was validated using the Modfold (http://www. reading.ac.uk/bioinf/ModFOLD/ModFOLD6_form. $\mathrm{html}$ ) server [13]. In order to investigate the structural changes in the NS proteins from the Iranian isolates compared to the templates, superimposition or structural alignment was performed using the Tm-align server (https://zhanglab.ccmb.med.umich.edu/TM-align/). The RMSD was calculated in Chimera 1.8.1 software.

\section{Results}

Virus samples from different patients have the expected 890-bp amplicons, and no signal was observed in the blank negative control (lane 11), indicating the absence of contamination and a successful RT-PCR procedure (Figure 1).

Table 3. influenza isolates from this study submitted to GenBank

\begin{tabular}{ccc}
\hline Number & Strain & Accession Numbers \\
\hline 1 & A/Tehran/A7109/2017 & MG663092.1 \\
\hline 2 & A/Tehran/A7108/2017 & MG663091.1 \\
\hline 3 & A/Tehran/A7107/2017 & MG663090.1 \\
\hline 4 & A/Tehran/A7106/2017 & MG663089.1 \\
\hline 5 & A/Tehran/A7105/2017 & MG663088.1 \\
6 & A/Tehran/A7104/2017 & MG663087.1 \\
\hline 7 & A/Tehran/A7103/2017 & MG663086.1 \\
8 & A/Tehran/A7101/2017 & MG663085.1 \\
\hline 9 & A/Tehran/Smo06/2015 & MG669373.1 \\
\hline 10 & A/Tehran/Smo07/2015 & MG669374.1 \\
11 & A/Tehran/Smo08/2015 & MG669375.1 \\
\hline 12 & A/Tehran/Smo09/2015 & MG669376.1 \\
\hline
\end{tabular}




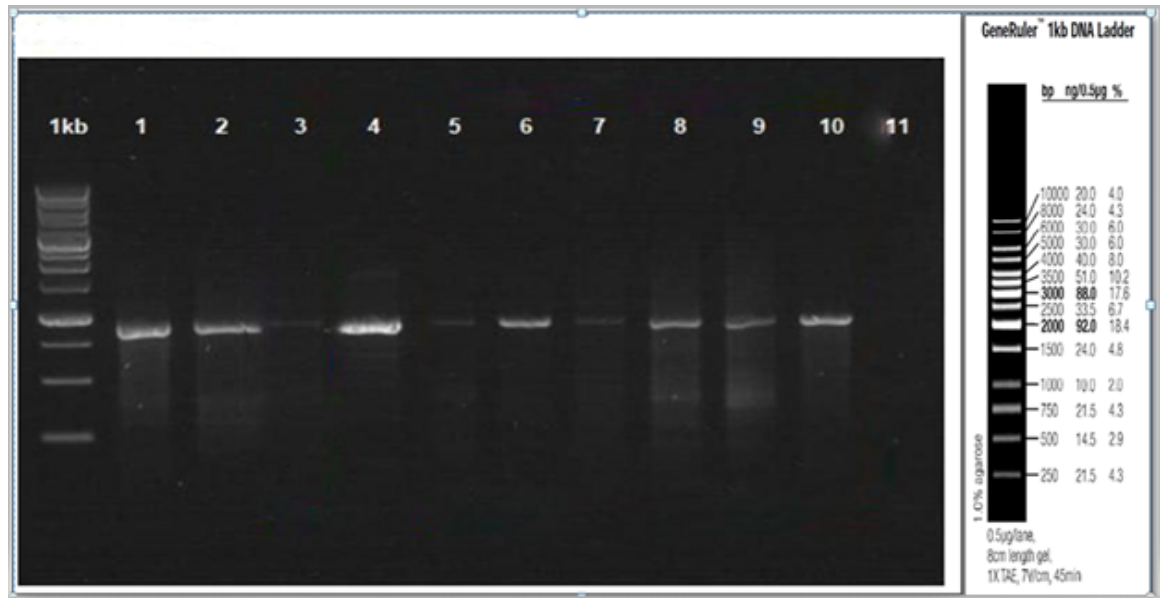

Figure 1. Agarose gel electrophoresis of the NS gene amplification

It shows the 890-bp amplicons from different patient samples. Lanes 1-10 positive samples; lane 11 blank negative control. Molecular weight marker: GeneRuler DNA ladder mix \#SM0313

The phylogenetic tree sproduced based on NS nucleotide and amino acid sequences suggest familiarity between all 12 NS sequences, including references (Figures 2 to 5). Results at both level of nucleic acid and amino acid revealed that all the subjected Iranian isolates from 2015 and 2017 outbreaks, except the A/Tehran/
SMO08/2015, were close to strains from Continental countries of Asia: (A/Tehran/1002/2016), Jeddah) A/ Jedah/2077/2015 ), India (A/India/Blora/2010)) as well as (A/Linkou/0046/2016) and (A/New York/01/2015) and (A/keelung/0039/2016) and (A/Passo Fando/LACENRS-1138/2016), with 99\% identity. Meanwhile,

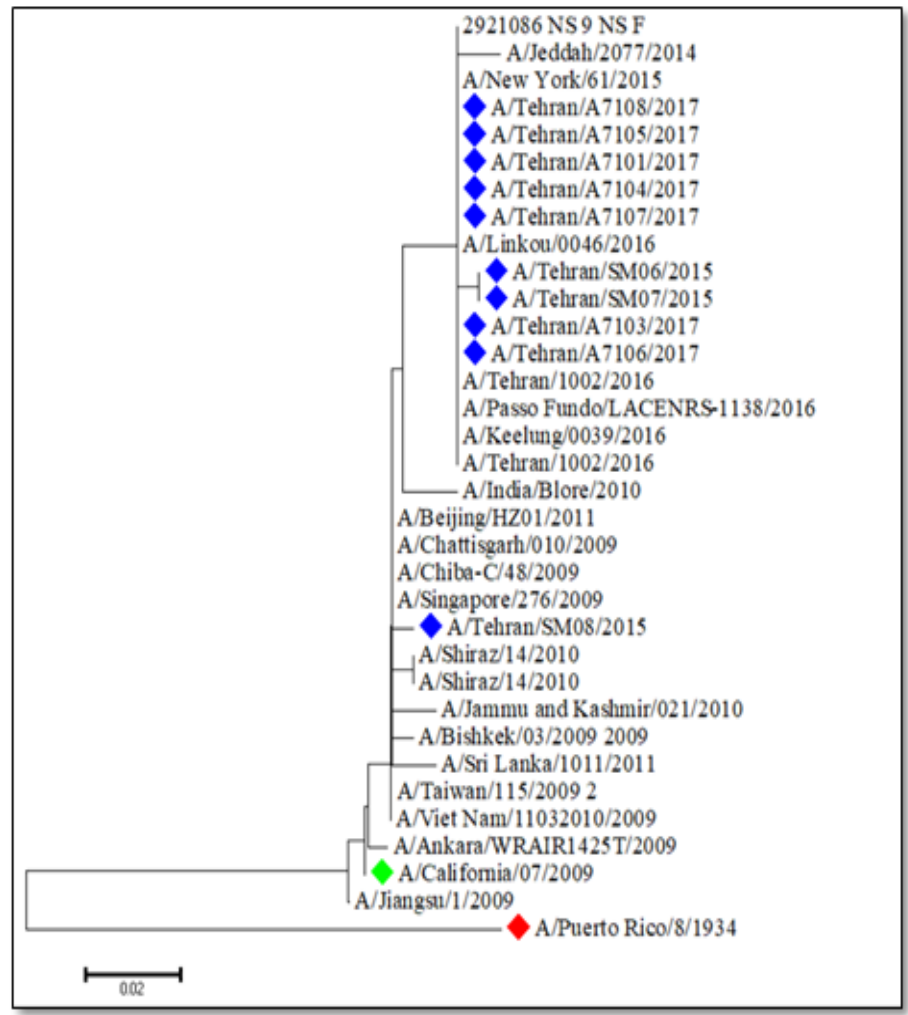

Figure 2. Phylogenetic tree of the NS1 gene of influenza A (H1N1) viruses using the neighbor-joining method

The vaccine strain [NC_026434.1/California/2009], the Iranian samples and the out-group strain (PR8) relevant to this study are highlighted by green and blue and red signs, respectively 


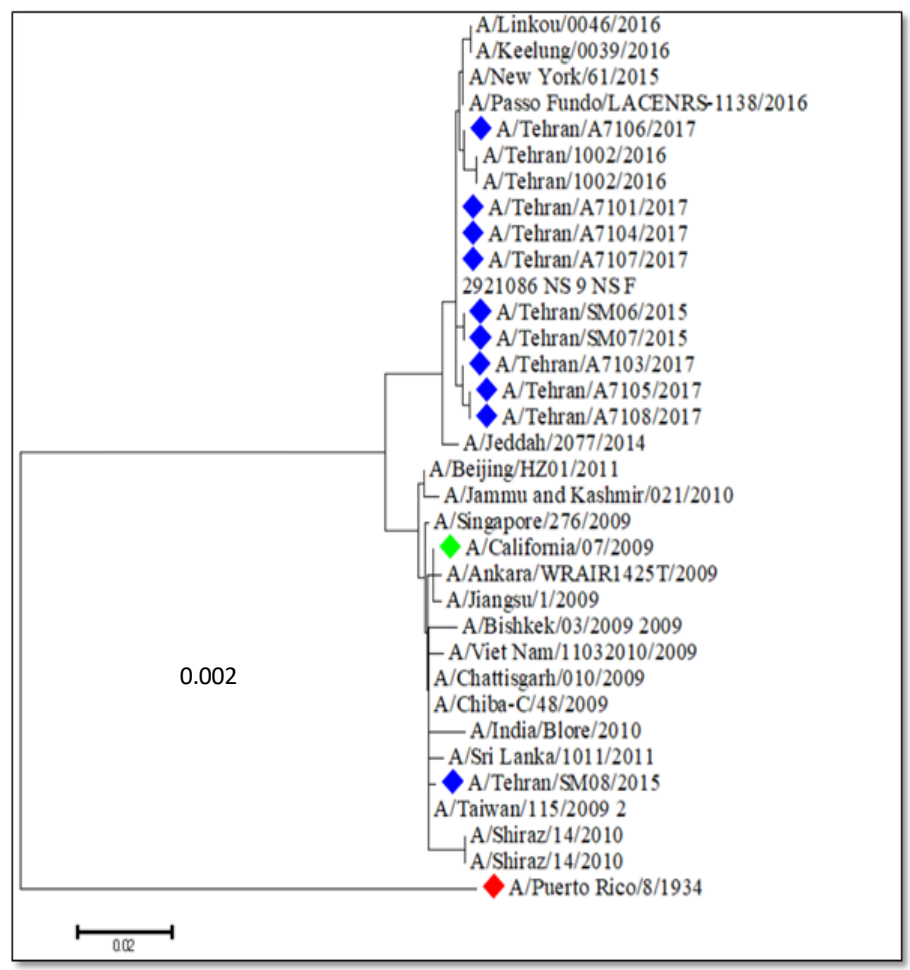

Figure 3. Phylogenetic tree of the NS1 proteins using the neighbor-joining method

The vaccine strain [NC_026434.1/California/2009], the Iranian samples and the out-group strain (PR8) relevant to this study are highlighted by green and blue and red signs, respectively.

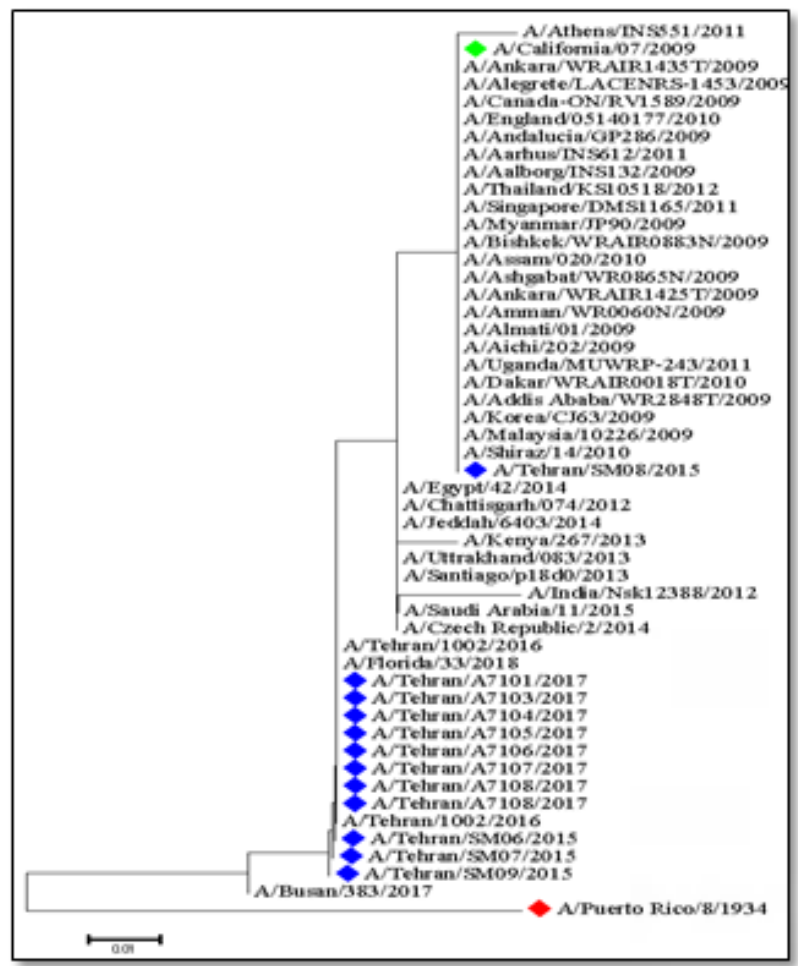

Figure 4. Phylogenetic tree of the NS2 gene of influenza A (H1N1) viruses using the neighbor-joining method

The vaccine strain [NC_026434.1/California/2009], the Iranian samples and the out-group strain (PR8) relevant to this study are highlighted by green and blue and red signs, respectively 


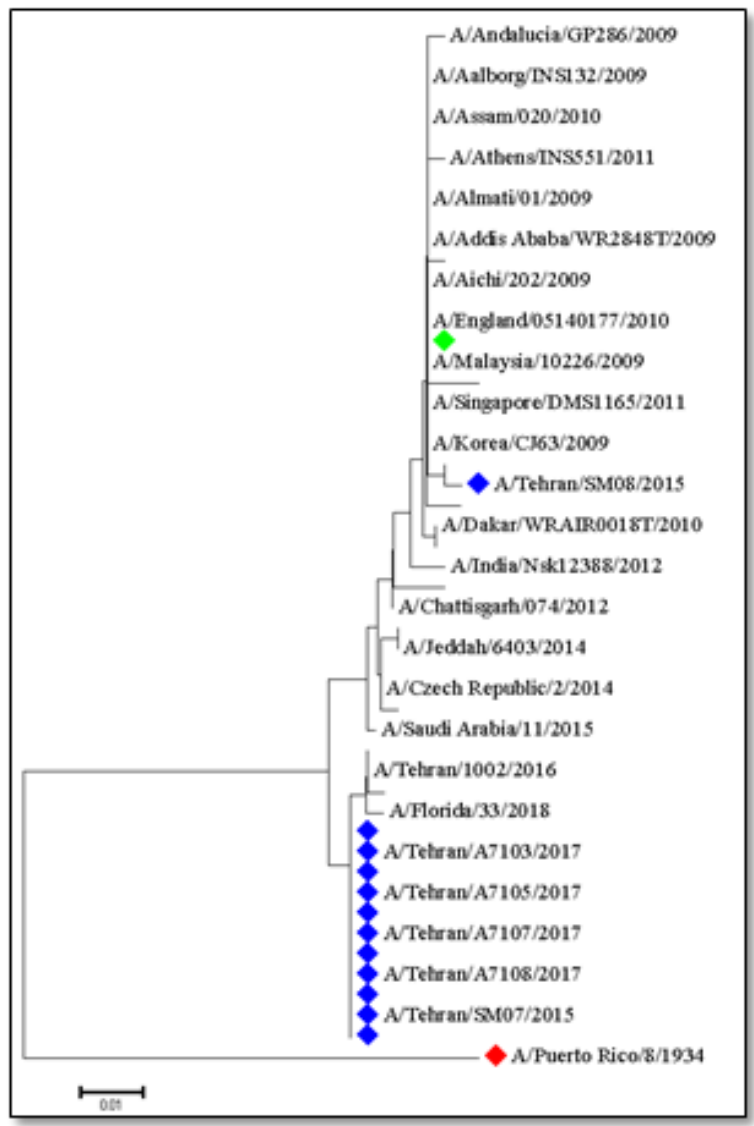

Figure 5. Phylogenetic tree of the NS2 proteins

The trees were constructed using neighbor joining method; The vaccine strain [NC_026434.1/California/2009], the Iranian samples and the out-group strain (PR8) relevant to this study are highlighted by green and blue and red signs, respectively

the exception was clustered with some strains from Iran and other Asian countries, such as Kazakhstan (A/Bishkek/03/2009) and Iran (A/Shiraz/14/2010, A/Jammu and Kashmir/021/2010, and A/Bishkek/03/2009).

In general, six substitutions were observed through the deduced Iranian NS1 and NS2 amino acid sequences (Table 4). One of the mutations (the E96D from the isolate A/Tehran/A7106/2017) was observed for the first time in this study. Prediction of three-dimensional structure of the Iranian NS1 and NS2 proteins in comparison with their counterpart references available in PDB showed a slight deviation in the functional domains (Figure 6 \& Figure 7).

Table 4. Observed mutations among NS1 and NS2 amino acid sequences

\begin{tabular}{ccc}
\hline NS1/NS2 & Sequence & Mutations \\
\hline E125D & All seq except A/Tehran/Smo08/2015 & NS1 \\
M83I & All seq except A/Tehran/Smo08/2015 & NS1 \\
L90I & All seq except A/Tehran/Smo08/2015 & NS1 \\
I123V & All seq & NS1 \\
N205S & All seq except A/Tehran/Smo08/2015 & Only \\
E96D & A/Tehran/A7106/2017 \\
\hline
\end{tabular}




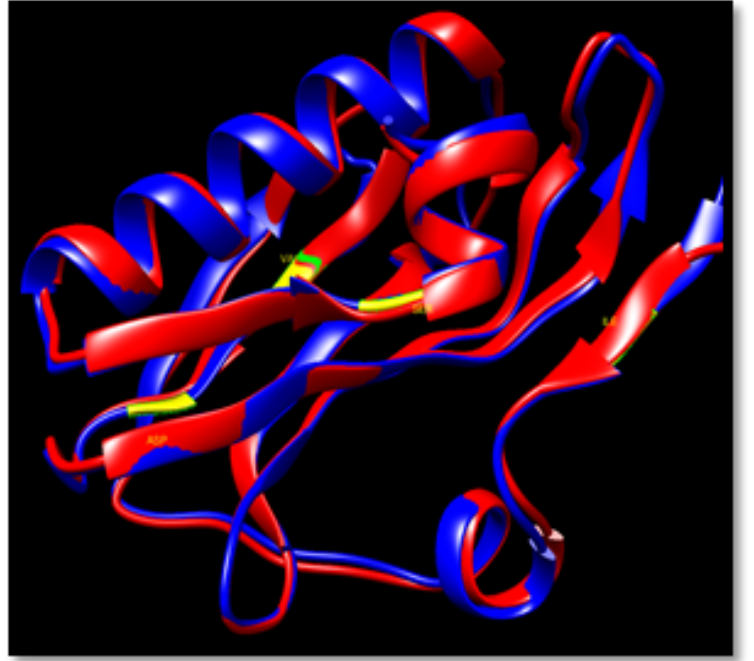

gRmm

Figure 6. Superimposition of the NS1 proteins from the study (blue) and the template (red)

Yellow arrows indicate the site of slight structural difference (RMSD: $0.448 \AA$ )

\section{Discussion}

Influenza A viruses are known to cause severe acute respiratory tract infections and represent a significant public health threat [14]. The influenza A/H1N1 virus caused pandemic flu in 2009 and is still circulating during the winter season in many countries [15]. The prevalence and influenza activity is monitored annually in Iran [16]. The use of viral genome sequence information and phylogenetic trees are often used to understand the evolution relationship, origin and spread of viruses.

The NS protein along with viral polymerase protein 2 and hemagglutinin are known to contribute to the virulence and host range restriction of influenza viruses. NS1 is considered a highly conserved influenza A gene; however, the species-specific evolution has been reported. In this study, the entire NS gene (encoding NS1 and NS2 proteins) sequencing was carried out on eleven influenza A (H1N1) samples from Iranian patients during the 2015 deadly flu outbreak and the 2017 seasonal outbreak. NS1 protein is functionally divided into two domains: the RBD (residues 1-73) and ED (residues 74-230).

Sequencing analysis from the NS1 gene reveal the $\mathrm{RBD}$ is more conserved compared to the ED. The ED is involved in interactions with the host factors, associated with cell signaling and immune response. The appearance of three mutations L90I, I123V, and N205S in ED has been observed in the most circulating $\mathrm{A}(\mathrm{H} 1 \mathrm{~N} 1)$

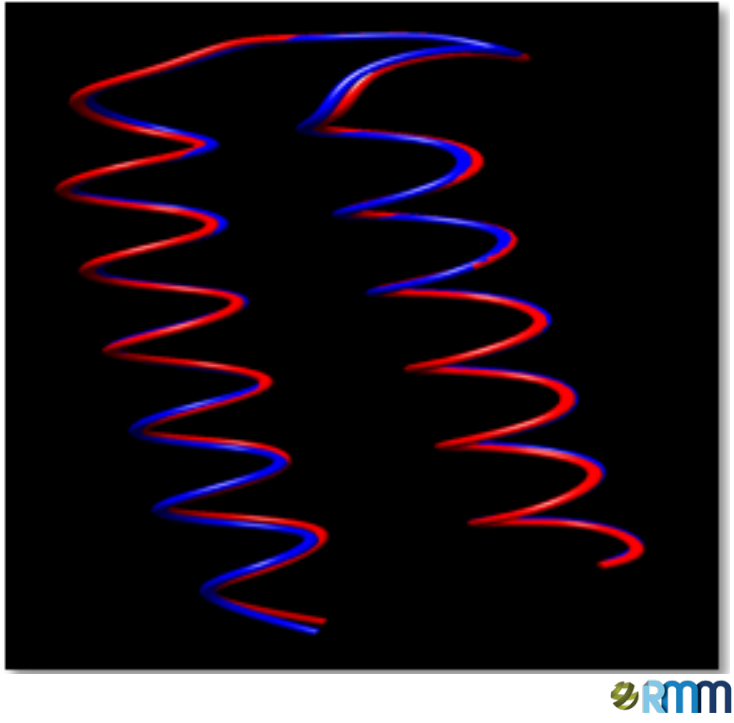

Figure 7. Superimposition of the NS2 proteins from this study (blue) and the template (red)

The RMSD value shows the structural difference between proteins NS2 and the template (RMSD: $0.615 \AA$ )

pdm09 viruses from 2010-2012 onwards and it seems to be stabilized as a feature [1].

It has been proven that L90I substitution in ED increases the virus replication power. Based on the deduced amino acid sequence, every sample from this study, except for the "A/Tehran/Smo08/2015", showed the I123V mutation in the ED. These mutations have been also reported in India during 2009-2013 [1]. E125D in NS1 is known to be one of the key substitutions involved in shutdown of host mRNA transport, restoring inherent disability of $\mathrm{A}(\mathrm{H} 1 \mathrm{~N} 1)$ pdm09 virus to efficiently control human cell gene expression. NS1 of all seasonal human influenza viruses (H1N1 and H3N2) contains D125 that interacts with cellular cleavage and polyadenylation factor 30 (CPSF30). Interaction with CPSF30 is absent in most animal-adapted strains. E125D as well as M83I substitution in the NEP protein were reported in the beginning of the 2015 epidemic season in Moscow and Saint Petersburg [14]. "A/Tehran/Smo08/2015" is also different from other Iranian samples by having E125D and M83I substitutions (Table 3).

As a conclusion, in terms of NS molecular features, almost all samples from this study (2015 and 2017) showed similar pattern of changes as observed in other geographical regions, although 2015 epidemic season in Iran was characterized by rapid increase of influenza, acute respiratory infection with high morbidity rate. None of mutations associated with increased virulence, such as R38, F103, and M106, were observed. Further 
studies on other genomic segments along with experimental proof are needed to understand the possible link between transmissibility or virulence.

\section{Ethical considerations}

\section{Compliance with ethical guidelines}

This article does not contain any studies involving human participants or animals performed by any of the authors. All ethical principles were considered in the study.

Funding

Malek-Ashtar University of Technology's Research Center for Biosciences and Biotechnology, Tehran, Iran.

\section{Authors contribution}

Designing and direcing the project: Zahra Asadollahi, Fatemeh Fotouhi and Behrokh Farahmand; Performing the experiments: Zahra Asadollahi and Samaneh Kouhestani; Writing the manuscript: Zahra Asadollahi and Farida Behzadian.

\section{Conflict of interest}

The authors declared no conflict of interest.

\section{References}

[1] Kumar S, Khare S, Saidullah B, Gandhoke I, Ram H Singh S. Appearance of L90I and N205S mutations in effector domain of NS1 Gene of pdm (09) H1N1 Virus from India during 2009-2013. Adv Virol. 2014; 2014:1-14. [DOI:10.1155/2014/861709] [PMID] [PMCID]

[2] Corbin A, Balvay L, Lina B, Rosa-calatrava M. The NS1 protein from influenza virus stimulates translation initiation by enhancing ribosome recruitment to mRNAs. J Mol Biol. 2017; 429(21):3334-52. [DOI:10.1016/j.jmb.2017.04.007] [PMID]

[3] Hale BG, Randall RE, Ortin J, Jackson D. The multifunctional NS1 protein of influenza A viruses. J Gen Virol. 2008; 89(10):2359-76. [DOI:10.1099/vir.0.2008/004606-0] [PMID]

[4] Grimm D, Staeheli P, Hufbauer M, Koerner I, Martínez-Sobrido L, Solórzano A, et al. Replication fitness determines high virulence of influenza A virus in mice carrying functional Mx1 resistance gene. Proc Natl Acad Sci. 2007; 104(16):680611. [DOI:10.1073/pnas.0701849104] [PMID] [PMCID]

[5] Dittmann J, Stertz S, Grimm D, Steel J, García-Sastre A, Haller $\mathrm{O}$, et al. Influenza A virus strains differ in sensitivity to the antiviral action of Mx-GTPase. J Virol. 2008; 82(7):3624-31. [DOI:10.1128/JVI.01753-07] [PMID] [PMCID]

[6] Hsiang TY, Zhou L, Krug RM. Roles of the phosphorylation of specific serines and threonines in the ns1 protein of human influenza a viruses. J Virol. 2012; 86(19):10370-6. [DOI:10.1128/JVI.00732-12] [PMID] [PMCID]
[7] Wang W, Riedel K, Lynch PA, Chein CY, Montelione GT, Krug RM. RNA binding by the novel helical domain of the influenza virus NS1 protein requires its dimer structure and a small number of specific basic amino acids. RNA. 1999; 5(2):195-205. [DOI:10.1017/S1355838299981621] [PMID] [PMCID]

[8] Lin D, Lan J, Zhang Z. Structure and function of the NS1 protein of influenza A virus. Acta Biochim Biophys Sin. 2007 39(3):155-62. [DOI:10.1111/j.1745-7270.2007.00263.x] [PMID]

[9] Dankar SK, Wang S, Ping J, Forbes NE, Keleta L, Li Y, et al. Influenza A virus NS1 gene mutations F103L and M106 increase replication and virulence. Virol J. 2011; 8(1):1-13. [DOI:10.1186/1743-422X-8-13] [PMID] [PMCID]

[10] Hoffmann E, Stech J, Guan Y, Webster RG, Perez DR. Universal primer set for the full-length amplification of all influenza A viruses. Arch Virol. 2001; 146(12):2275-89. [DOI:10.1007/s007050170002] [PMID]

[11] Saitou N, Nei M. The neighbor-joining method: A new method for reconstructing phylogenetic trees. Molecular biol Evolut. 1987; 4(4):406-25.

[12] Pieper U, Eswar N, Davis FP, Braberg H, Madhusudhan MS, Rossi A, et al. MODBASE: A database of annotated comparative protein structure models and associated resources. Nucleic Acids Res. 2006; 34(suppl 1):D291-5. [DOI:10.1093/ nar/gkj059] [PMID] [PMCID]

[13] Maghrabi AH, McGuffin LJ. ModFOLD6: An accurate web server for the global and local quality estimation of 3D protein models. Nucleic Acids Res. 2017; 45(W1):W416-21. [DOI:10.1093/nar/gkx332] [PMID] [PMCID]

[14] Klenk HD, Wagner R, Heuer D, Wolff T. Importance of hemagglutinin glycosylation for the biological functions of influenza virus. Virus Res. 2002; 82(1-2):73. [DOI:10.1016/ S0168-1702(01)00389-6]

[15] Jhung MA, Swerdlow D, Olsen SJ, Jernigan D, Biggerstaff M, Kamimoto L, et al. Epidemiology of 2009 pandemic influenza A (H1N1) in the United States. Clin Infect Dis. 2011; 52(1):S13-26. [DOI:10.1093/cid/ciq008] [PMID]

[16] Mohebbi A, Fotouhi F, Jamali A, Yaghobi R, Farahmand B Mohebbi R. Molecular epidemiology of the hemagglutinin gene of prevalent influenza virus A/H1N1/pdm09 among patient in Iran. Virus Res; 2019(259):38-45. [DOI:10.1016/j. virusres.2018.10.001] [PMID] 